\title{
Looking forward to millicharged dark sectors at the LHC
}

\author{
Saeid Foroughi-Abari๑, ${ }^{1, *}$ Felix Kling $\odot,{ }^{2, \dagger}$ and Yu-Dai Tsai $\odot^{3, \hbar}$ \\ ${ }^{1}$ Department of Physics and Astronomy, University of Victoria, \\ Victoria, British Columbia V8P 5C2, Canada \\ ${ }^{2}$ SLAC National Accelerator Laboratory, 2575 Sand Hill Road, Menlo Park, California 94025, USA \\ ${ }^{3}$ Theory Department, Fermi National Accelerator Laboratory (Fermilab), Batavia, Illinois 60510, USA
}

(Received 1 January 2021; accepted 22 July 2021; published 16 August 2021)

\begin{abstract}
We identify what may be the world's most sensitive location to search for millicharged particles in the $10 \mathrm{MeV}$ to $100 \mathrm{GeV}$ mass range: the forward region at the LHC. We propose constructing a scintillatorbased experiment, FORward MicrOcharge SeArch (FORMOSA) in this location. FORMOSA can discover millicharged particles in a large and unexplored region of parameter space, including millicharged strongly interacting dark matter (mSIDM) candidates that cannot be probed by ground-based direct-detection experiments. The newly proposed LHC Forward Physics Facility (FPF) provides an ideal structure to host the full FORMOSA experiment.
\end{abstract}

DOI: $10.1103 /$ PhysRevD.104.035014

\section{INTRODUCTION}

Searching for millicharged particles, or MCPs, provides an empirical test of charge quantization [1] as well as predictions from well motivated UV theories, including grand unified theories (GUT) $[2,3]$ and string theory $[4,5]$ ). MCPs can also arise as a low-energy consequence of a theory with a massless kinetic-mixing dark photon [6]. Recently, the consideration of MCPs as dark matter (DM) [7-9] and the connection to the explanation to the EDGES anomaly [10-14] rekindle the experimental MCP program.

Within the past few decades, the search for MCPs has encompassed terrestrial experiments at fixed target and collider facilities, as well as astrophysical and cosmological observations [15-25]. A dedicated experiment, milliQan [22], was proposed at LHC that would detect MCPs produced by proton collisions using scintillator-based detectors. Later, FerMINI [26] with a similar setup was considered for proton fixed-target and neutrino experiments, primarily targeting Fermilab proton beamlines or CERN SPS beam. Recently, this idea has motivated a proposal at J-PARC proton fixed-target facilities [27].

In general, there is great promise in using experiments at the intersection of the high-energy and high-intensity frontiers to study dark-sector or long-lived particles.

\footnotetext{
*saeidf@uvic.ca

†felixk@slac.stanford.edu

¥ytsai@fnal.gov
}

Published by the American Physical Society under the terms of the Creative Commons Attribution 4.0 International license. Further distribution of this work must maintain attribution to the author(s) and the published article's title, journal citation, and DOI. Funded by SCOAP.
Traditionally such searches are done either in the LHC transverse region or at proton fixed-target experiments $[25,26,28-51]$. However, we note that the production rates for dark-sector particles available in the forward direction of high-energy colliders are comparable to those achieved at beam dump experiments, so one can view the detectors located at the LHC forward regions as very energetic beamdump experiments given the high statistics one can accumulate in this region [52]. The LHC's forward region can be regarded as a true intersection between high energy and high intensity: this is where one can get a high flux of lowmass dark sector particles through direct production and meson decays. However, until recently, this region has been neglected.

In this paper, we consider two scenarios featuring millicharged particles in the LHC forward physics region. First, we consider installing a minimal MCP detector in a tunnel next to the current FASER experiment (see the left panel of Fig. 1 for more details), which we call FORMOSA-I for convenience. Second, we consider constructing a full-size milliQan-type detector in the Forward Physics Facility (FPF, an expanded UJ12 hall [53]), referred to as FORMOSA-II. One can also consider moving the proto-milliQan detector [54] to the forward physics region, but given the higher beam-related background rate compared to the transverse region [55] a more detailed study of the sensitivity is required.

\section{LOCATION}

We propose locating FORMOSA in the far-forward direction, close to the beam collision axis, where it can benefit from an enhanced MCP production cross section compared to the transverse direction. 

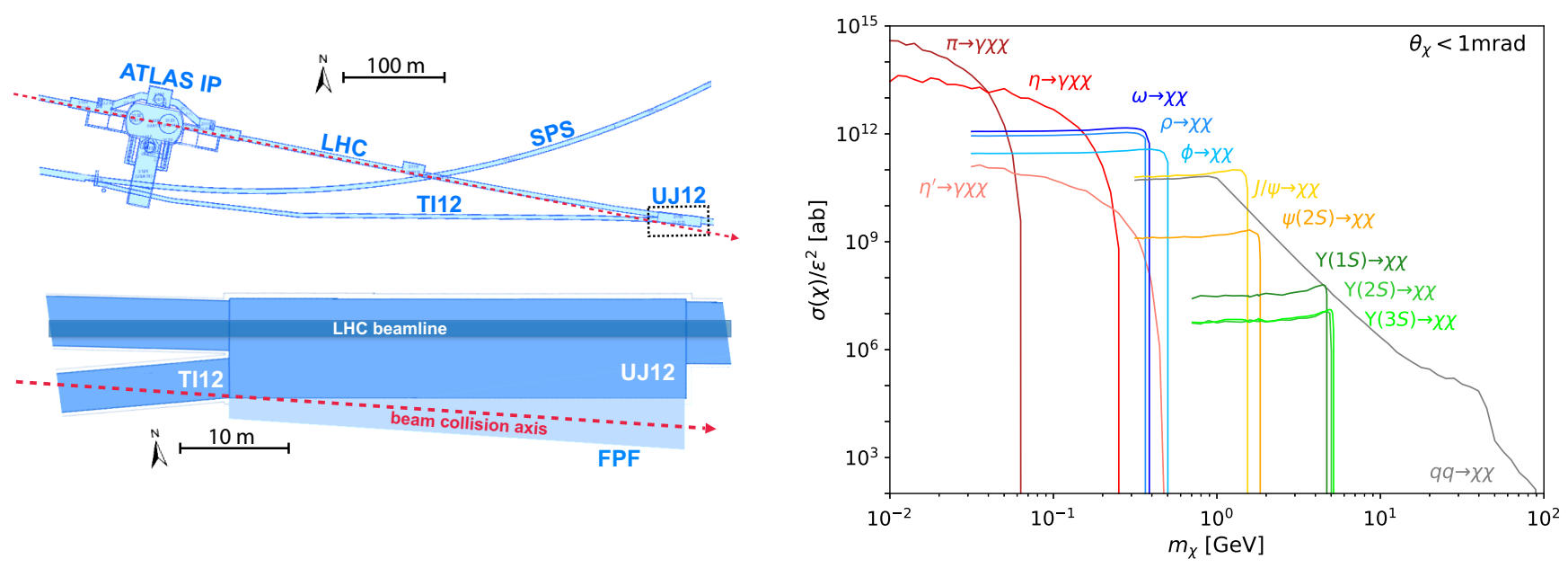

FIG. 1. Left: the proposed location of FORMOSA in the cavern UJ12 or side tunnel TI12 (blue) close to the beam collision axis (red). The Forward Physics Facility (FPF) extension is shown as a light-blue area. Right: production cross section of MCP in the forward direction, $\theta_{\chi}<1 \mathrm{mrad}$, for different production modes and MCP masses.

A suitable location is available roughly $500 \mathrm{~m}$ downstream from the ATLAS interaction point in the cavern UJ12 or the tunnel TI12, shown in the left panel of Fig. 1. One can also consider the nearly symmetrical TI18 and UJ18 on the opposite side of ATLAS. During Run 3 of the LHC, TI12 will host the FASER experiment to search for long-lived particles [51,52,55-61], and study neutrino interactions [62,63], with continuations being proposed for the HL-LHC era [64,65]. For this reason, TI12 and UJ12 are equipped with lighting, power, stairs, and support structures to safely transport detector components around the LHC. Recently, it has also been proposed to enlarge the UJ12 cavern to create a FPF, which could house FORMOSA and other forward experiments [53].

TI12 and UJ12 are shielded from the ATLAS IP by the forward LHC infrastructure, which consists of magnets and absorbers, as well as $100 \mathrm{~m}$ of rock. Particle fluxes and radiation levels have been simulated using FLUKA [66] and validated experimentally $[55,63]$, indicating that the particle fluxes at this location are low.

\section{MCP PRODUCTION}

We study MCP $\chi$ with electric charge $Q_{\chi}$, mass $m_{\chi}$ and define $\epsilon \equiv Q_{\chi} / e$. The small electric charge can come from directly introducing a tiny $U(1)$ hypercharge to $\chi$. It can also be generated by a massless dark photon, kinetically mixed with SM, that couples to $\chi$ and induces the millicharge of $\chi$ in a convenient basis [6,67].

We perform a dedicated Monte Carlo study to estimate the flux of MCP produced at the LHC. The different production channels and their corresponding production cross sections in the forward direction, within $\theta_{\chi}<1 \mathrm{mrad}$ of the beam collision axis, are summarized in the right panel of Fig. 1.

If the MCP is light, it is primarily produced in both pseudoscalar meson decays such as $\pi^{0} \rightarrow \gamma \chi \chi$ and vector meson decays such as $\omega, J / \psi, \Upsilon \rightarrow \chi \chi$. We generate the spectra of light mesons using EPOS-LHC [68]. The spectra of the charmonium and bottomonium states are simulated using PYTHIA8 [69], which we have calibrated with their spectra as measured at LHCb [70-72]. More details on the simulation and validation of forward meson production at the LHC and their decays into MCPs can be found in Appendix.

Heavy MCPs are primarily produced in partonic scattering $q q \rightarrow \chi \chi$. We simulate this Drell-Yan production mode using MADGRAPH5 [73] and PYTHIA 8. To ensure that the PDFs are well defined, we require the invariant mass of the MCP pair to be larger than $2 \mathrm{GeV}$, which leads to a constant MCP production cross sections for $m_{\chi}<1 \mathrm{GeV}$.

It is important to note that particle production rates are enhanced in the forward direction. According to Feynman scaling arguments [74], in the limit where $m_{\chi} \ll p_{T}$, MCP production is approximately flat in pseudorapidity, $d N / d \eta \approx$ constant. Comparing the flux of MCPs going through a $1 \mathrm{~m} \times 1 \mathrm{~m}$ area at a transverse location (T) similar to milliQan, and a forward location (F) about $500 \mathrm{~m}$ downstream and off-set by $2 \mathrm{~m}$ from the beam axis, we find $N_{\mathrm{F}} / N_{\mathrm{T}} \sim \Delta \eta_{F} / \Delta \eta_{T} \times \Delta \phi_{\mathrm{F}} / \Delta \phi_{\mathrm{T}} \sim 250$, indicating that the forward particle flux is indeed strongly enhanced.

\section{DETECTOR AND SIGNATURE}

We will consider a minimal detector (FORMOSA-I) and a full MCP detector (FORMOSA-II). For FORMOSA-I we consider a setup similar to the milliQan demonstrator with size $0.2 \mathrm{~m} \times 0.2 \mathrm{~m} \times 4 \mathrm{~m}$, which consists of 4 layers each containing 16 scintillator bars coupled to a PMT. FORMOSA-II would be a $1 \mathrm{~m} \times 1 \mathrm{~m} \times 4 \mathrm{~m}$ array consisting of 4 layers of 400 scintillator bars. In our analysis we place FORMOSA-I in the UJ12/TI12 hall and 2 meters offaxis, and we take FORMOSA-II to be on-axis and located in the proposed FPF (expanded UJ12 hall). 
When an MCP traverses the detector, photoelectrons (PE) are produced from ionization energy deposition within each stack of scintillator. The average number of PEs collected with detection efficiency $\varepsilon_{\text {det }}$ in a scintillator bar with density $\rho_{s}$ and length $L_{s}$ can be estimated as $\bar{N}_{\mathrm{PE}} \approx \varepsilon_{\mathrm{det}} \rho_{s} L_{s} \times\langle-d E / d x\rangle \times Y_{\gamma}$, where the mean rate of energy deposition $\langle-d E / d x\rangle$ scales as $\epsilon^{2}$ and is a function of the MCPs energy [75]. $Y_{\gamma} \sim 1.1 \times 10^{4} \mathrm{MeV}^{-1}$ is the photon yield deposited in the typical plastic scintillators [76]. Like the milliQan collaboration [23], we assume a detection efficiency of $\epsilon \approx 10 \%$.

For FORMOSA, we search for a quadruple coincidence of hits with $\bar{N}_{\mathrm{PE}} \geq 1$ in each stack within a $20 \mathrm{~ns}$ time window. The probability of detecting a MCP follows the Poisson distribution, $P_{\operatorname{det}}=\left(1-\exp \left(-\bar{N}_{\mathrm{PE}}\right)\right)^{4}$. Considering the number of MCPs passing through the detector $N_{\chi}$, the total number of signal events is $N_{\chi} \cdot P_{\text {det }}$.

\section{BACKGROUND}

The potential background sources of an MCP detector at the forward physics region can be classified as beam related and beam unrelated. According to the proto-milliQan study [54], in the transverse region, the beam-unrelated background dominates, and it can be controlled by adding an additional layer to the original three-layer millQan design. However, in the forward region at the LHC considered in this paper, we find that the beam-related background could be much more important. The background reduction strategy and the choices of PMTs need to be reconsidered accordingly. We will first review the beam unrelated background and its reduction strategy, and then discuss the beam-related background.

The beam unrelated background of FORMOSA can come from cosmic muons and dark current, and their combined coincident signatures. The cosmic muons interacting with the cavern walls generate a spray of electrons and gamma rays. The subsequent shower causing scintillation in the detector is a significant background source for the proto-milliQan detector [54]. In addition, due to the random emission of thermal electrons from the photocathode, dark current pulses can be produced in each PMT. These two sources of detector background lead to the similar signature to MCPs.

Several techniques have been developed to reduce these beam-unrelated background events $[22,23]$. These strategies can be summarized as requiring multiple-coincidence as a detection signature; implementing a veto of large-PE events; shifting or enlarging the middle detector array (to eliminate coincident low-PE pulses caused by energetic muons scraping through the surface of the scintillator layers); and considering a dead-time veto of the afterpulses. In addition to scintillator bars, the milliQan prototype design has considered components such as the scintillator panels and four scintillator slabs along the length of the detector for further reduction of backgrounds [54]. The scintillator panels are used as shields for the bars from the top and sides to reject backgrounds due to cosmic muon showers. The slabs provide time information, shielding from neutron radiation, and help to veto deposits due to beam and cosmic muons passing the bars. As demonstrated by the milliQan collaboration, the beam unrelated background can be reduced to nearly zero with all these background reduction strategies.

The quadruple coincidence requirement has the advantage of lowering the dark-current background rate by a factor of $\mathcal{O}\left(10^{-4}\right)$ compared to the original milliQan design, which is expected to have $\sim 300$ dark current background events per year $[23,26]$. The same conclusion taking into account other beam unrelated background is also shown in [77].

A new challenge arises for the dedicated MCP search in the forward physics region, given a large flux of highenergy muons from the beam collisions. For FORMOSA-I, we consider a location inside the cavern UJ12, where a muon rate $\lesssim 1 \mathrm{~Hz} / \mathrm{cm}^{2}$ can be achieved [62] (one can consider the same muon rate for FORMOSA-II). These energetic muons (and the secondary particles they produce inside the detector) can cause large pulses in the PMT. One can implement online-vetos of large-PE pulses to avoid the readout deadtime and ensure a high signal efficiency.

In addition, afterpulses, which can appear with a delay time of a few $\mu$ s after the initial pulse, could become sources of background events. These smaller pulses can appear correlated in the $\tau$ time window. Thus, they may be indistinguishable from the small PE events and cannot be vetoed by the large PE cuts. However, the rate of the afterpulses with a delay time of $\delta t \gtrsim 10 \mu$ s drops below the dark current rate for most of the PMTs [78,79]. We can therefore remove the afterpulse background using a veto: assuming one muon every $100 \mu$ s for FORMOSA-II and an afterpulsing duration of $\sim 10 \mu \mathrm{s}$, roughly $10 \%$ of the data needs to be vetoed, resulting in a live-time efficiency of $\sim 0.9$. For FORMOSA-I, this is a much smaller issue since it has a smaller detector area and thus a lower total muon rate. Considering better PMTs with reduced afterpulse duration can improve the live-time efficiencies for both FORMOSA-I and II.

Interactions of high-energy neutrinos produced at the LHC with the FORMOSA detector provide another beamrelated background source. According to [63], we estimate $\mathcal{O}\left(10^{6}\right)$ interactions with FORMOSA during the HL-LHC. These interactions cause energetic events with a very large number of $\mathrm{PE}$ throughout the detector, which is very different from the MCP signal. Furthermore, neutrinos interacting with the material surrounding FORMOSA can produce particles that form a hadronic shower. In a $2.5 \mathrm{~m}$ volume of rock in front of FORMOSA (corresponding to $\sim 10 \lambda_{\text {int }}$ which a hadronic shower should be mostly absorbed) $\mathcal{O}\left(10^{6}\right)$ neutrino interactions is expected during 

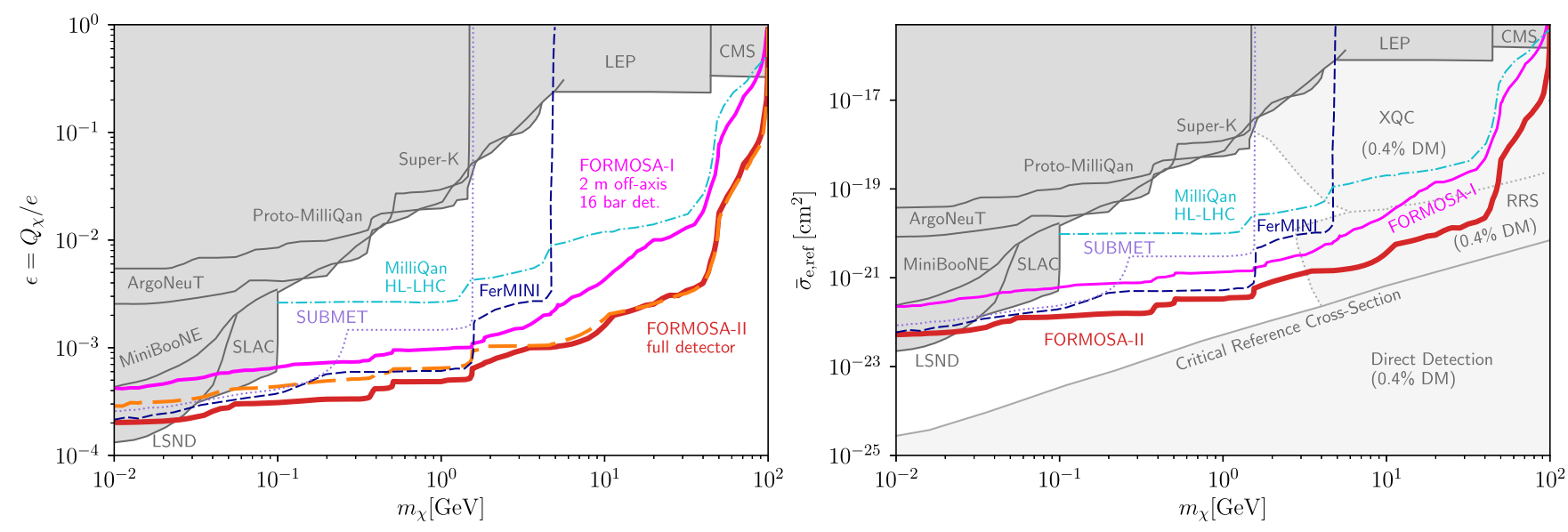

FIG. 2. Sensitivity of FORMOSA in the MCP parameter space (left) and mSIDM parameter space (right) alongside existing accelerator constraints (dark gray), direct-detection experiments (light gray, assuming 0.4\% DM abundance for the direct-detection experiments) and other proposals (dashed lines). FORMOSA-II $2 \mathrm{~m}$ off-axis is plotted orange and dashed. See text for details.

the HL-LHC. This corresponds to a rate of $\mathcal{O}(1)$ interaction per minute, which is subleading compared to the expected rate of muons passing through the detector.

Other beam-related background sources come from beam-gas and beam halo collisions with the beam pipe, which require special attention. Based on a FLUKA study ${ }^{1}$ performed for the FASER experiment $[55,66]$, we expect that particles produced in proton losses and beam-gas collisions near TI12/UJ12 would typically point toward the direction of the nearby LHC beam pipe rather than the ATLAS IP. This lead us to suggest (i) additional shielding and (ii) vetoes around the detector, combined with pointing the long axis of FORMOSA toward the IP, to reduce possible backgrounds associated with such particles. Note that the FLUKA simulations do not include any nonbeam backgrounds such as cosmic ray muons, and thus are insufficient to quantitatively estimate the background rate for the new detector. As we mentioned, one can install the proto-milliQan at this location to conduct an in-situ study of the background to address this issue.

\section{SENSITIVITY}

In the left panel of Fig. 2, we present the projected sensitivity reaches of FORMOSA-I and II in terms of the $\mathrm{MCP}$ mass $m_{\chi}$ and charge ratio $\epsilon \equiv Q_{\chi} / e$, assuming an integrated luminosity of $3 \mathrm{ab}^{-1}$ at the HL-LHC. We require 3 signal events, and a 0.9 live-time efficiency. The choice of 3 events is based on the discussions in the background section that the beam-unrelated background can be reduced

\footnotetext{
${ }^{1}$ This simulation from Refs. [55,66] were performed for TI18/ UJ18 location, which is nearly identical to TI12/UJ12 and located on the opposite side of ATLAS. The results presented in Ref. [55] assumes LHC Run 3 conditions with a luminosity of $2 \times 10^{34} \mathrm{~cm}^{-2} \mathrm{sec}^{-1}$, while the original FLUKA study presented in Ref. [66] assumed HL-LHC conditions with a luminosity of $5 \times 10^{34} \mathrm{~cm}^{-2} \mathrm{sec}^{-1}$.
}

to a negligible level with the requirement of quadruple coincidence. The line corresponding to FORMOSA-I placed $2 \mathrm{~m}$ off-axis is colored magenta. FORMOSA-II on-axis ( $2 \mathrm{~m}$ off-axis) is plotted red (orange and dashed).

We plot the existing constraints as gray-shaded regions. These include bounds from SLAC [16], LEP [17,80], CMS [81,82], LSND and MiniBooNE [25], ArgoNeuT at Fermilab [83], proto-milliQan at LHC [54], and the constraint on cosmic-ray produced MCPs from Super-K [84]. For comparison, we also show the sensitivity projections from the full milliQan experiment at the $14 \mathrm{TeV}$ LHC with $3 \mathrm{ab}^{-1}$ integrated luminosity [23], the proposed FerMINI experiment at LBNF/DUNE with a beam energy of $120 \mathrm{GeV}$ and $10^{21}$ POT (also considered for NuMI/ MINOS hall and the sensitivity reach is similar) [26], and the proposed SUBMET experiment assuming $10^{22} \mathrm{POT}$ at the $30 \mathrm{GeV}$ proton beam at J-PARC [27].

Based on our analysis, one can see that FORMOSA-I, a minimal detector to be placed in UJ12, would provide a better sensitivity reach in comparison to the full milliQan run. As discussed above, this is due to an enhanced flux of MCP in the forward region, in comparison to that of the transverse region. Up to $\mathcal{O}\left(10^{6}\right)$ signal events at $m_{\chi}=$ $1 \mathrm{GeV}$ near the proto-milliQan bound are possible. We also find that with $300 \mathrm{fb}^{-1}$ (1/10 of the full luminosity of the HL-LHC), the FORMOSA-I has a better sensitivity than the full HL-LHC milliQan for $m_{\chi} \lesssim 10 \mathrm{GeV}$.

Also note that, although we derive our sensitivity projections based on the luminosity of HL-LHC, it could be possible to employ a similar setup already during Run 3 of the LHC. In particular, one could install the protomilliQan detector in the TI12 cavern and perform a test run for FORMOSA to better understand the detector environment and experimental challenges.

We further show that FORMOSA-II provides leading sensitivity projections in a large window of MCP mass from $100 \mathrm{MeV}$ to $100 \mathrm{GeV}$, exceeding the reaches of other similar 
proposals. We also see that, even if the detector is placed a few meters off the beam axis, the sensitivity would not be strongly affected. This allows us to place FORMOSA inside the existing cavern UJ12.

\section{MILLICHARGED STRONGLY INTERACTING DM}

MCPs in this region of parameter space can account for a fraction of the dark matter (DM) abundance, but cannot be detected by the direct-detection experiments when the cross section is larger than certain critical values (derived in $[85,86])$. The ambient DM (DM in our local galaxy with Standard distribution and velocity dependence [87]) with a substantial cross section with Standard Model (SM) particles can lose most of its kinetic energy through interactions with SM particles. For some model parameters, the DM particles lose most of their energy and hence cannot be detected by ground-based direct detection experiments after interacting with the atmospheric particles and the crust. These DM particles are generally referred to as strongly interacting DM (SIDM) [88-90]. In [84-86], an unconstrained region of parameter space is identified, which can be referred to as a millicharged SIDM (mSIDM) window, and FORMOSA can provide strong sensitivity in this parameter space.

In the right panel of Fig. 2, we show our results in terms of $m_{\chi}$ and the conventional "reference cross section" $\bar{\sigma}_{e, \text { ref }}=16 \pi \alpha^{2} \epsilon^{2} \mu_{\chi e}^{2} / q_{d, \text { ref }}^{4}$. Here, $q_{d \text {,ref }}$ is chosen to be the typical momentum transfer in $\chi-e$ scattering for semiconductor or noble-liquid targets (taken to be $\alpha m_{e}$ [86]) and $\mu_{\chi e}$ is the reduced mass of the electron and $\chi$.

We include bounds from terrestrial direct detection experiments [85,86], XQC [91] and RRS [88]. We do not plot the constraints based on the millicharged DM accelerated by astrophysical sources [92-95], as they require additional assumptions beyond local DM properties. When presenting the bounds, we follow Ref. [86] and assume $0.4 \%$ of the DM to be MCP to avoid strong cosmological constraints [96-98]. In addition, the CMB constraint is preventing the DM from being composed of majority of MCP, and $0.4 \%$ of DM being MCP is allowed by the current constraint (see, e.g., [99]). For different assumptions of DM being MCP, the existing constraints from direct-detection experiments would be different. It is also demonstrated that $0.4 \%$ of DM being MCP could potentially explain the EDGES anomaly, although subjecting to other constraints (see, e.g., $[11,100]$ ).

We show that FORMOSA can help cover a large part of the millicharged DM region that is previously unconstrained. Our study also demonstrates two strong advantages of accelerator probes of DM in general: (i) the accelerator probes are not sensitive to the material's attenuation, given that the particles produced from beam interactions have large kinetic energy (unlike the ambient DM, which has much lower kinetic energy), and (ii) the accelerator constraints are independent of the fractional composition of DM (unlike the direct-detection or cosmological probes [96-98]).

\section{CONCLUSION}

In this letter, we propose a new $\mathrm{MCP}$ search in the forward region of the LHC ATLAS interaction point. FORMOSA, a milliQan-like experiment placed $\sim 500 \mathrm{~m}$ downstream from ATLAS, would take advantage of enhanced MCP production in the forward direction and can provide leading sensitivity to MCPs in the $10 \mathrm{MeV}$ to $100 \mathrm{GeV}$ mass window.

We also find that, unlike the current milliQan location, beam-related backgrounds associated with the sizable flux of forward muons, such as PMT afterpulses, become important in the forward direction. This motivates additional detector design considerations, such as the use of PMTs with low afterpulse duration or the application of vetoes to control the afterpulse background.

In addition to MCPs, FORMOSA has the potential to search for other beyond standard model (BSM) particles, such as heavy neutrinos and DM with electric dipoles [101-104]. Furthermore, FORMOSA's location in the far forward direction allows further extensions of its physics objectives. For example, one would expect about $10^{6}$ neutrino interactions in the FORMOSA-II detector, providing additional opportunities for neutrino physics $[62,65,105]$ and forward particle production measurements, and indicating a physics potential of FORMOSA and the Forward Physics Facility that remains to be explored.

\section{ACKNOWLEDGMENTS}

We would like to thank Matthew Citron, Patrick de Niverville, Jonathan Feng, and Christopher Hill for useful discussions. We also thank Matthew Citron, Jonathan Feng and Adam Ritz for valuable and detailed comments on the draft. We are grateful to the authors and maintainers of many open-source software packages, including CRMC [106], EPOS-LHC [68], JUPYTER notebooks [107], MADGRAPH5 [73], Matplotlib [108], NumPy [109], РyНерMC [110], PYTHIA8 [69] and Scikit-HEP [111]. F. K. is supported by U.S. Department of Energy Grant No. DE-AC0276SF00515. The work of S. F. was supported in part by NSERC, Canada. Part of this document was prepared by Y.-D. T. using the resources of the Fermi National Accelerator Laboratory (Fermilab), a U.S. Department of Energy, Office of Science, HEP User Facility. Fermilab is managed by Fermi Research Alliance, LLC (FRA), acting under Contract No. DE-AC02-07CH11359.

\section{APPENDIX: MCP PRODUCTION IN MESON DECAYS}

If the MCP $\chi$ is sufficiently light, $m_{\chi} \lesssim 5 \mathrm{GeV}$, it can be produced in the decay of SM mesons $M$. This requires a 

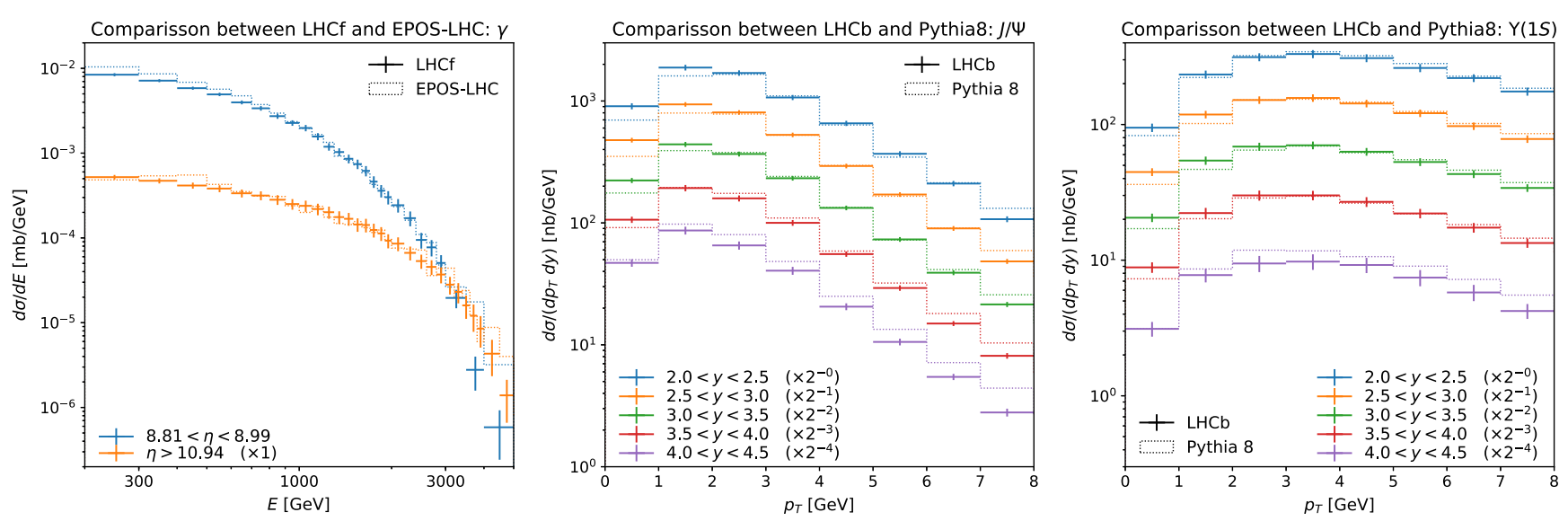

FIG. 3. Validation of forward meson production. Forward photon production predicted by EPOS-LHC (left), $J / \psi$ production (center) and $\Upsilon(1 S)$ production predicted by PYTHIA8 compared to measurements by LHCf [112] and LHCb [70,71] at 13 TeV LHC.

reliable description of forward particle production, best validated with or tuned to available data.

The production of light mesons, $M=\pi^{0}, \eta, \eta^{\prime}, \omega, \rho$ and $\phi$, is simulated using EPOS-LHC [68], as implemented in the simulation package CRMC [106], which is a dedicated Monte-Carlo generator designed to describe minimum bias hadronic interactions at both particle colliders and cosmic ray experiments. In the left panel of Fig. 3 we compare the predicted energy spectra of far forward photons (mainly from $\pi^{0}$ decay) produced in $13 \mathrm{TeV}$ collisions to those measured at LHCf [112], and find good agreement over the full spectrum.

In addition, we simulate the production of charmonium and bottomonium using PYTHIA8 [69]. Since the default setup of PYTHIA8 tends to overestimate their production rate at small transverse momenta $p_{T}$, we use the predefined SuppressSmallPT user hook to suppresses the production rate by a factor

$$
\left(\frac{p_{T}^{2}}{k^{2} p_{T 0}^{2}+p_{T}^{2}}\right)^{2}\left(\frac{\alpha_{S}\left(k^{2} p_{T 0}^{2}+Q_{\mathrm{ren}}^{2}\right)}{\alpha_{S}\left(Q_{\mathrm{ren}}^{2}\right)}\right)^{n}
$$

where $p_{T 0}$ is the same energy-dependent dampening scale as used for multiparton interactions and $Q_{\text {ren }}$ is the renormalization scale. Good agreement between the simulation and measurements at LHCb [70-72] are obtained for $k=0.35$ and $n=3$, as shown in the center and right panel of Fig. 3.

In the next step, we use a MC simulation to subsequently decay the mesons into MCPs $\chi$. The pseudoscalar mesons $M=\pi^{0}, \eta, \eta^{\prime}$ can undergo the 3-body decay $M \rightarrow \gamma \chi \bar{\chi}$. Following Refs. [48,113], the differential branching fraction for this process is given by

$$
\begin{aligned}
& \frac{d \mathrm{BR}(M \rightarrow \gamma \chi \chi)}{d s d \cos \theta}=\frac{\epsilon^{2} \alpha}{4 \pi s}\left[1-\frac{s}{m_{M}^{2}}\right]^{3}\left[1-\frac{4 m_{\chi}^{2}}{s}\right]^{\frac{1}{2}} \\
& \times\left[2-\left(1-\frac{4 m_{\chi}^{2}}{s}\right) \sin ^{2} \theta\right] \times \mathrm{BR}(M \rightarrow \gamma \gamma)
\end{aligned}
$$

where $s=\left(p_{\chi}+p_{\bar{\chi}}\right)^{2}$ is the invariant mass of the off-shell photon producing the MCP pair and $\theta$ is the angle between the momentum of $\chi$ in the off-shell photon's rest frame and the boost direction of the off-shell photon.

In addition, the vector mesons $M=\rho, \omega, \phi, J / \psi, \psi(2 S)$ and $\Upsilon(n S)$ can decay directly into a pair of MCPs, $M \rightarrow \chi \bar{\chi}$. Following Ref. [26], the corresponding branching fraction is given by

$\frac{\operatorname{BR}(M \rightarrow \chi \bar{\chi})}{\operatorname{BR}(M \rightarrow e e)}=\epsilon^{2} \frac{\left(m_{M}^{2}+2 m_{\chi}^{2}\right)\left(m_{M}^{2}-4 m_{\chi}^{2}\right)^{1 / 2}}{\left(m_{M}^{2}+2 m_{e}^{2}\right)\left(m_{M}^{2}-4 m_{e}^{2}\right)^{1 / 2}}$.
[1] P. A. M. Dirac, Quantized singularities in the electromagnetic field, Proc. R. Soc. A 133, 60 (1931); Erratum, Proc. R. Soc. A 133, 278 (1931).

[2] J. C. Pati and A. Salam, Unified lepton-hadron symmetry and a gauge theory of the basic interactions, Phys. Rev. D 8, 1240 (1973).
[3] H. Georgi, The state of the artgauge theories, AIP Conf. Proc. 23, 575 (1975).

[4] X.-G. Wen and E. Witten, Electric and magnetic charges in superstring models, Nucl. Phys. B261, 651 (1985).

[5] G. Shiu, P. Soler, and F. Ye, Milli-Charged Dark Matter in Quantum Gravity and String Theory, Phys. Rev. Lett. 110, 241304 (2013). 
[6] B. Holdom, Two U(1)'s and $\epsilon$ charge shifts, Phys. Lett. 166B, 196 (1986).

[7] D. E. Brahm and L. J. Hall, U(1)-prime dark matter, Phys. Rev. D 41, 1067 (1990).

[8] J. L. Feng, M. Kaplinghat, H. Tu, and H.-B. Yu, Hidden charged dark matter, J. Cosmol. Astropart. Phys. 07 (2009) 004.

[9] J. M. Cline, Z. Liu, and W. Xue, Millicharged atomic dark matter, Phys. Rev. D 85, 101302(R) (2012).

[10] J. D. Bowman, A. E. E. Rogers, R. A. Monsalve, T. J. Mozdzen, and N. Mahesh, An absorption profile centred at 78 megahertz in the sky-averaged spectrum, Nature (London) 555, 67 (2018).

[11] R. Barkana, Possible interaction between baryons and dark-matter particles revealed by the first stars, Nature (London) 555, 71 (2018).

[12] A. Berlin, N. Blinov, G. Krnjaic, P. Schuster, and N. Toro, Dark matter, millicharges, axion and scalar particles, gauge bosons, and other new physics with LDMX, Phys. Rev. D 99, 075001 (2019).

[13] T. R. Slatyer and C.-L. Wu, Early-Universe constraints on dark matter-baryon scattering and their implications for a global 21 cm signal, Phys. Rev. D 98, 023013 (2018).

[14] H. Liu, N. J. Outmezguine, D. Redigolo, and T. Volansky, Reviving millicharged dark matter for 21-cm cosmology, Phys. Rev. D 100, 123011 (2019).

[15] M. I. Dobroliubov and A. Yu. Ignatiev, Millicharged Particles, Phys. Rev. Lett. 65, 679 (1990).

[16] A. A. Prinz et al., Search for Millicharged Particles at SLAC, Phys. Rev. Lett. 81, 1175 (1998).

[17] S. Davidson, S. Hannestad, and G. Raffelt, Updated bounds on millicharged particles, J. High Energy Phys. 05 (2000) 003.

[18] E. Golowich and R. W. Robinett, Limits on millicharged matter from beam dump experiments, Phys. Rev. D 35, 391 (1987).

[19] K. S. Babu, T. M. Gould, and I. Z. Rothstein, Closing the windows on MeV Tau neutrinos, Phys. Lett. B 321, 140 (1994).

[20] S. N. Gninenko, N. V. Krasnikov, and A. Rubbia, Search for millicharged particles in reactor neutrino experiments: A Probe of the PVLAS anomaly, Phys. Rev. D 75, 075014 (2007).

[21] R. Agnese et al. (CDMS Collaboration), First Direct Limits on Lightly Ionizing Particles with Electric Charge Less Than e/6, Phys. Rev. Lett. 114, 111302 (2015).

[22] A. Haas, C. S. Hill, E. Izaguirre, and I. Yavin, Looking for milli-charged particles with a new experiment at the LHC, Phys. Lett. B 746, 117 (2015).

[23] A. Ball et al., A Letter of intent to install a milli-charged particle detector at LHC P5, arXiv:1607.04669.

[24] S. I. Alvis et al. (Majorana Collaboration), First Limit on the Direct Detection of Lightly Ionizing Particles for Electric Charge as Low as e/1000 with the Majorana Demonstrator, Phys. Rev. Lett. 120, 211804 (2018).

[25] G. Magill, R. Plestid, M. Pospelov, and Y.-D. Tsai, Millicharged Particles in Neutrino Experiments, Phys. Rev. Lett. 122, 071801 (2019).
[26] K. J. Kelly and Y.-D. Tsai, Proton fixed-target scintillation experiment to search for millicharged dark matter, Phys. Rev. D 100, 015043 (2019).

[27] S. Choi et al., Letter of intent: Search for sub-millicharged particles at J-PARC, arXiv:2007.06329.

[28] B. Batell, M. Pospelov, and A. Ritz, Exploring portals to a hidden sector through fixed targets, Phys. Rev. D 80, 095024 (2009).

[29] R. Essig, R. Harnik, J. Kaplan, and N. Toro, Discovering new light states at neutrino experiments, Phys. Rev. D 82, 113008 (2010).

[30] P. deNiverville, M. Pospelov, and A. Ritz, Observing a light dark matter beam with neutrino experiments, Phys. Rev. D 84, 075020 (2011).

[31] Y. Kahn, G. Krnjaic, J. Thaler, and M. Toups, DAEDALUS and dark matter detection, Phys. Rev. D 91, 055006 (2015).

[32] P. deNiverville, M. Pospelov, and A. Ritz, Light new physics in coherent neutrino-nucleus scattering experiments, Phys. Rev. D 92, 095005 (2015).

[33] S. Gardner, R. J. Holt, and A. S. Tadepalli, New prospects in fixed target searches for dark forces with the SeaQuest experiment at Fermilab, Phys. Rev. D 93, 115015 (2016).

[34] E. Izaguirre, G. Krnjaic, and M. Pospelov, MeV-scale dark matter deep underground, Phys. Rev. D 92, 095014 (2015).

[35] M. Pospelov and Y.-D. Tsai, Light scalars and dark photons in Borexino and LSND experiments, Phys. Lett. B 785, 288 (2018).

[36] L. Darm, S. Rao, and L. Roszkowski, Light dark Higgs boson in minimal sub-GeV dark matter scenarios, J. High Energy Phys. 03 (2018) 084.

[37] G. Magill, R. Plestid, M. Pospelov, and Y.-D. Tsai, Dipole portal to heavy neutral leptons, Phys. Rev. D 98, 115015 (2018).

[38] A. Berlin, S. Gori, P. Schuster, and N. Toro, Dark sectors at the Fermilab SeaQuest Experiment, Phys. Rev. D 98, 035011 (2018).

[39] J. R. Jordan, Y. Kahn, G. Krnjaic, M. Moschella, and J. Spitz, Signatures of pseudo-Dirac dark matter at highintensity neutrino experiments, Phys. Rev. D 98, 075020 (2018).

[40] P. deNiverville and C. Frugiuele, Hunting sub-GeV dark matter with the $\mathrm{NO} \nu \mathrm{A}$ near detector, Phys. Rev. D 99, 051701(R) (2019).

[41] E. Bertuzzo, S. Jana, P. A. N. Machado, and R. Zukanovich Funchal, Dark Neutrino Portal to Explain MiniBooNE excess, Phys. Rev. Lett. 121, 241801 (2018).

[42] E. Bertuzzo, S. Jana, P. A. N. Machado, and R. Zukanovich Funchal, Neutrino masses and mixings dynamically generated by a light dark sector, Phys. Lett. B 791, 210 (2019).

[43] P. Ballett, S. Pascoli, and M. Ross-Lonergan, U(1)' mediated decays of heavy sterile neutrinos in MiniBooNE, Phys. Rev. D 99, 071701(R) (2019).

[44] C. A. Argelles, M. Hostert, and Y.-D. Tsai, Testing New Physics Explanations of MiniBooNE Anomaly at Neutrino Scattering Experiments, Phys. Rev. Lett. 123, 261801 (2019).

[45] B. Batell, A. Freitas, A. Ismail, and D. McKeen, Probing light dark matter with a hadrophilic scalar mediator, Phys. Rev. D 100, 095020 (2019). 
[46] C. A. Argüelles et al., White paper on new opportunities at the next-generation neutrino experiments (Part 1: BSM neutrino physics and dark matter), Rep. Prog. Phys. 83, 124201 (2020).

[47] Y.-D. Tsai, P. deNiverville, and M. X. Liu, The HighEnergy Frontier of the Intensity Frontier: Closing the Dark Photon, Inelastic Dark Matter, and Muon g-2 Windows, Phys. Rev. Lett. 126, 181801 (2021).

[48] V. De Romeri, K. J. Kelly, and P. A. Machado, DUNEPRISM sensitivity to light dark matter, Phys. Rev. D 100, 095010 (2019).

[49] B. Dbrich, J. Jaeckel, and T. Spadaro, Light in the beam dump. Axion-like particle production from decay photons in proton beam-dumps, J. High Energy Phys. 05 (2019) 213.

[50] L. Buonocore, C. Frugiuele, and P. deNiverville, Hunt for sub-GeV dark matter at neutrino facilities: A survey of past and present experiments, Phys. Rev. D 102, 035006 (2020).

[51] A. Ariga et al. (FASER Collaboration), FASER's physics reach for long-lived particles, Phys. Rev. D 99, 095011 (2019).

[52] J. L. Feng, I. Galon, F. Kling, and S. Trojanowski, ForwArd Search ExpeRiment at the LHC, Phys. Rev. D 97, 035001 (2018).

[53] J. L. Feng, F. Kling et al., Forward physics facility, https:// doi.org/10.5281/zenodo.4009641.

[54] A. Ball et al., Search for millicharged particles in protonproton collisions at $\sqrt{s}=13 \mathrm{TeV}$, Phys. Rev. D 102, 032002 (2020).

[55] A. Ariga et al. FASER Collaboration, Technical proposal for FASER: ForwArd Search ExpeRiment at the LHC, arXiv:1812.09139.

[56] J. L. Feng, I. Galon, F. Kling, and S. Trojanowski, Dark Higgs bosons at the ForwArd Search ExpeRiment, Phys. Rev. D 97, 055034 (2018).

[57] F. Kling and S. Trojanowski, Heavy neutral leptons at FASER, Phys. Rev. D 97, 095016 (2018).

[58] J. L. Feng, I. Galon, F. Kling, and S. Trojanowski, Axionlike particles at FASER: The LHC as a photon beam dump, Phys. Rev. D 98, 055021 (2018).

[59] A. Berlin and F. Kling, Inelastic dark matter at the LHC lifetime frontier: ATLAS, CMS, LHCb, CODEX-b, FASER, and MATHUSLA, Phys. Rev. D 99, 015021 (2019).

[60] A. Ariga et al. (FASER Collaboration), Letter of intent for FASER: ForwArd Search ExpeRiment at the LHC, arXiv:1811.10243.

[61] F. Kling and S. Trojanowski, Looking forward to test the KOTO anomaly with FASER, Phys. Rev. D 102, 015032 (2020).

[62] H. Abreu et al. (FASER Collaboration), Detecting and studying high-energy collider neutrinos with FASER at the LHC, Eur. Phys. J. C 80, 61 (2020).

[63] H. Abreu et al. (FASER Collaboration), Technical proposal: FASERnu, arXiv:2001.03073.

[64] FASER Collaboration, FASER 2: Forward Search Experiment at the HL LHC. https://www.snowmass21 .org/docs/files/summaries/EF/SNOWMASS21-EF9_EF6NF3_NF6-RF6_RF0-CF7_CF0-AF5_AF0_FASER2-038 .pdf.
[65] FASER Collaboration, FASERv2: A Forward Neutrino Experiment at the HL LHC. https://www.snowmass21 .org/docs/files/summaries/NF/SNOWMASS21-NF10_ NF6-EF6_EF9-IF0_FASERnu2-006.pdf.

[66] CERN Sources, Targets, and Interactions Group, M. Sabate-Gilarte, F. Cerutti, and A. Tsinganis, Characterization of the radiation field for the FASER experiment, Technical Report, CERN, 2018.

[67] L. B. Okun, Limits of electrodynamcis: Paraphotons? Zh. Eksp. Teor. Fiz. 83, 892 (1982) [Sov. Phys. JETP 56, 502 (1982)].

[68] T. Pierog, I. Karpenko, J. M. Katzy, E. Yatsenko, and K. Werner, EPOS LHC: Test of collective hadronization with data measured at the CERN Large Hadron Collider, Phys. Rev. C 92, 034906 (2015).

[69] T. Sjstrand, S. Ask, J. R. Christiansen, R. Corke, N. Desai, P. Ilten, S. Mrenna, S. Prestel, C. O. Rasmussen, and P. Z. Skands, An Introduction to PYTHIA8.2, Comput. Phys. Commun. 191, 159 (2015).

[70] R. Aaij et al. (LHCb Collaboration), Measurement of forward $J / \psi$ production cross-sections in $p p$ collisions at $\sqrt{s}=13 \mathrm{TeV}$, J. High Energy Phys. 10 (2015) 172; Erratum, J. High Energy Phys. 05 (2017) 063.

[71] R. Aaij et al. (LHCb Collaboration), Measurement of $\Upsilon$ production in $p p$ collisions at $\sqrt{s}=13 \mathrm{TeV}$, J. High Energy Phys. 07 (2018) 134; Erratum, J. High Energy Phys. 05 (2019) 076.

[72] R. Aaij et al. (LHCb Collaboration), Measurement of $\psi(2 S)$ production cross-sections in proton-proton collisions at $\sqrt{s}=7$ and $13 \mathrm{TeV}$, Eur. Phys. J. C 80, 185 (2020).

[73] J. Alwall, R. Frederix, S. Frixione, V. Hirschi, F. Maltoni, O. Mattelaer, H. S. Shao, T. Stelzer, P. Torrielli, and M. Zaro, The automated computation of tree-level and nextto-leading order differential cross sections, and their matching to parton shower simulations, J. High Energy Phys. 07 (2014) 079.

[74] J. F. Grosse-Oetringhaus and K. Reygers, Charged-particle multiplicity in proton-proton collisions, J. Phys. G 37, 083001 (2010).

[75] M. Tanabashi et al. (Particle Data Group Collaboration), Review of particle physics, Phys. Rev. D 98, 030001 (2018).

[76] Saint-Gobain, Plastic scintillators, https://www.crystals saint-gobain.com/products/organic-scintillation-materials, accessed: 2016-02-12.

[77] A. Ball et al. (MilliQan Collaboration), Sensitivity to millicharged particles in future proton-proton collisions at the LHC, arXiv:2104.07151.

[78] S. Aiello et al. (KM3NeT Collaboration), Characterisation of the Hamamatsu photomultipliers for the KM3NeT neutrino telescope, J. Instrum. 13, P05035 (2018).

[79] P.-A. Amaudruz et al. (DEAP Collaboration), In-situ characterization of the Hamamatsu R5912-HQE photomultiplier tubes used in the DEAP-3600 experiment, Nucl. Instrum. Methods Phys. Res., Sect. A 922, 373 (2019).

[80] R. Akers et al. (OPAL Collaboration), Search for heavy charged particles and for particles with anomalous charge in $e^{+} e^{-}$collisions at LEP, Z. Phys. C 67, 203 (1995). 
[81] S. Chatrchyan et al. (CMS Collaboration), Search for fractionally charged particles in $p p$ collisions at $\sqrt{s}=7$ TeV, Phys. Rev. D 87, 092008 (2013).

[82] J. Jaeckel, M. Jankowiak, and M. Spannowsky, LHC probes the hidden sector, Phys. Dark Universe 2, 111 (2013).

[83] R. Acciarri et al. (ArgoNeuT Collaboration), Improved Limits on Millicharged Particles Using the ArgoNeuT Experiment at Fermilab, Phys. Rev. Lett. 124, 131801 (2020).

[84] R. Plestid, V. Takhistov, Y.-D. Tsai, T. Bringmann, A. Kusenko, and M. Pospelov, New constraints on millicharged particles from cosmic-ray production, Phys. Rev. D 102, 115032 (2020).

[85] M. S. Mahdawi and G. R. Farrar, Constraints on dark matter with a moderately large and velocity-dependent DM-nucleon cross-section, J. Cosmol. Astropart. Phys. 10 (2018) 007.

[86] T. Emken, R. Essig, C. Kouvaris, and M. Sholapurkar, Direct detection of strongly interacting sub-GeV dark matter via electron recoils, J. Cosmol. Astropart. Phys. 09 (2019) 070.

[87] J. Bovy and S. Tremaine, On the local dark matter density, Astrophys. J. 756, 89 (2012).

[88] J. Rich, R. Rocchia, and M. Spiro, A search for strongly interacting dark matter, Phys. Lett. B 194, 173 (1987).

[89] G. D. Starkman, A. Gould, R. Esmailzadeh, and S. Dimopoulos, Opening the window on strongly interacting dark matter, Phys. Rev. D 41, 3594 (1990).

[90] G. D. Starkman, A. Gould, R. Esmailzadeh, and S. K. Dimopoulos, Opening the window on strongly interacting dark matter, Phys. Rev. D 41, 3594 (1990).

[91] A. L. Erickcek, P. J. Steinhardt, D. McCammon, and P. C. McGuire, Constraints on the interactions between dark matter and baryons from the x-ray quantum calorimetry experiment, Phys. Rev. D 76, 042007 (2007).

[92] P.-K. Hu, A. Kusenko, and V. Takhistov, Dark cosmic rays, Phys. Lett. B 768, 18 (2017).

[93] D. Dunsky, L. J. Hall, and K. Harigaya, CHAMP cosmic rays, J. Cosmol. Astropart. Phys. 07 (2019) 015.

[94] J.-T. Li and T. Lin, Dynamics of millicharged dark matter in supernova remnants, Phys. Rev. D 101, 103034 (2020).

[95] L. Chuzhoy and E. W. Kolb, Reopening the window on charged dark matter, J. Cosmol. Astropart. Phys. 07 (2009) 014.

[96] S. L. Dubovsky, D. S. Gorbunov, and G. I. Rubtsov, Narrowing the window for millicharged particles by CMB anisotropy, Pis'ma Zh. Eksp. Teor. Fiz. 79, 3 (2004) [JETP Lett. 79, 1 (2004)].

[97] A. D. Dolgov, S. L. Dubovsky, G. I. Rubtsov, and I. I. Tkachev, Constraints on millicharged particles from Planck data, Phys. Rev. D 88, 117701 (2013).
[98] E. D. Kovetz, V. Poulin, V. Gluscevic, K. K. Boddy, R. Barkana, and M. Kamionkowski, Tighter limits on dark matter explanations of the anomalous EDGES $21 \mathrm{~cm}$ signal, Phys. Rev. D 98, 103529 (2018).

[99] T. R. Slatyer, Indirect dark matter signatures in the cosmic dark ages. I. Generalizing the bound on s-wave dark matter annihilation from Planck results, Phys. Rev. D 93, 023527 (2016).

[100] A. Berlin, D. Hooper, G. Krnjaic, and S. D. McDermott, Severely Constraining Dark Matter Interpretations of the 21-cm Anomaly, Phys. Rev. Lett. 121, 011102 (2018).

[101] M. Sher and J. Stevens, Detecting a heavy neutrino electric dipole moment at the LHC, Phys. Lett. B 777, 246 (2018).

[102] X. Chu, J. Pradler, and L. Semmelrock, Light dark states with electromagnetic form factors, Phys. Rev. D 99, 015040 (2019).

[103] M. Frank, M. de Montigny, P.-P. A. Ouimet, J. Pinfold, A. Shaa, and M. Staelens, Searching for heavy neutrinos with the MoEDAL-MAPP detector at the LHC, Phys. Lett. B 802, 135204 (2020).

[104] X. Chu, J.-L. Kuo, and J. Pradler, Dark sector-photon interactions in proton-beam experiments, Phys. Rev. D 101, 075035 (2020).

[105] W. Bai, M. Diwan, M. V. Garzelli, Y. S. Jeong, and M. H. Reno, Far-forward neutrinos at the Large Hadron Collider, J. High Energy Phys. 06 (2020) 032.

[106] C. Baus, T. Pierog, and R. Ulrich, Cosmic Ray Monte Carlo (CRMC),. https://web.ikp.kit.edu/rulrich/crmc.html.

[107] T. Kluyver et al., Jupyter notebooks_A publishing format for reproducible computational workflows, in Positioning and Power in Academic Publishing: Players, Agents and Agendas (IOS Press, 2016), pp. 87-90, https://eprints .soton.ac.uk/403913/.

[108] J. D. Hunter, Matplotlib: A 2d graphics environment, Comput. Sci. Eng. 9, 90 (2007).

[109] T. Oliphant, NumPy: A Guide to NumPy (Trelgol publishing, USA, 2006), http://www.numpy.org/.

[110] A. Buckley, Pyhepmc 1.0, 2018. https://doi.org/10.5281/ zenodo.1304136.

[111] E. Rodrigues, The Scikit-HEP project, EPJ Web Conf. 214, 06005 (2019).

[112] O. Adriani et al. (LHCf Collaboration), Measurement of forward photon production cross-section in proton-proton collisions at $\sqrt{s}=13 \mathrm{TeV}$ with the LHCf detector, Phys. Lett. B 780, 233 (2018).

[113] K. Jodlowski, F. Kling, L. Roszkowski, and S. Trojanowski, Extending the reach of FASER, MATHUSLA, and SHiP towards smaller lifetimes using secondary particle production, Phys. Rev. D 101, 095020 (2020). 